\title{
Association of pro-inflammatory cytokines with QCT-measured bone mineral density and its gender difference in a Chinese population - a pilot study
}

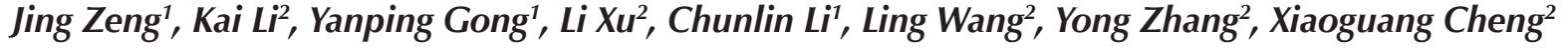 \\ ${ }^{1}$ Department of Geriatric Endocrinology, Chinese People's Liberation Army General Hospital, National Clinical Research Centre \\ for Geriatric Disease, Beijing, China \\ ${ }^{2}$ Department of Radiology, Beijing Jishuitan Hospital, Beijing, China
}

\begin{abstract}
Introduction: The primary objective of this study was to explore the association of pro-inflammatory cytokines with quantitative computed tomography (QCT)-measured bone mineral density (BMD) and its sexual difference in a Chinese population.

Material and methods: In a cross-sectional study, a total of 85 participants aged 48-85 years were recruited and classified into three groups: normal bone mass with BMD $>120 \mathrm{mg} / \mathrm{cm}^{3}$, osteopaenia with BMD $80-120 \mathrm{mg} / \mathrm{cm}^{3}$, and osteoporosis with BMD $<80 \mathrm{mg} / \mathrm{cm}^{3}$. ANOVA analysis and multivariable ordinal logistic regression was used to estimate the associations of interest.

Results: The proportions of osteoporosis, osteopaenia, and normal BMD were $29.41 \%$ (25), 37.65\% (32), and 32.94\% (28), respectively. Males had the highest proportion of osteopaenia while females had osteoporosis ( $\mathrm{p}<0.01)$. In contrast with the null findings in males, significant statistical difference was observed in females. Compared to the normal BMD, the OR for increasing IL-6 level of decreasing BMD groups (osteoporosis and/or osteopaenia) was 9.50 (95\% CI: 1.44-62.80). TNF- $\alpha$ and IL-17 tended to show an increased risk (OR > 1) but did not reach significance. Current OR result cannot confirm these relationships of IL- $1 \beta$, and ORs of PAI- 1 and MCP- 1 are too close to 1 to determine the practical meaning.

Conclusions: IL-6 is an obviously independent risk factor of QCT-measured osteoporosis in Chinese females. Sex hormones and fat-related factors are important confounders in the research of osteoimmunology. (Endokrynol Pol 2019; 70 (3): 248-254)

Key words: pro-inflammatory cytokines; QCT; bone mineral density; cross-sectional study
\end{abstract}

\section{Introduction}

Osteoporosis is prevalent in the middle-aged and older populations with more than 200 million individuals affected worldwide [1]. In China, about 69.44 million people aged 50 years or over suffer from osteoporosis, while 210 million have low bone mass, and the number of osteoporosis cases is expected to rise to 286.6 million by 2020 [2]. As one of the consequences of osteoporosis, fractures are becoming a severe disease burden to society. The medical costs of 2015, 2035, and 2050 for primary osteoporosis fracture (wrist, vertebral body, and hip) will be as high as 1.1 billion, 1.9 billion, and 2.4 billion dollars, respectively [3]. Hence, active efforts to prevent osteoporosis are necessary.

Multiple risk factors have been recognised to contribute to the occurrence and development of osteoporosis, including sex, age, obesity, diabetes, and trauma [4]. Several basic studies show that innate and adaptive immunity cells produce inflammatory cytokines that not only perpetuate inflammation but also may activate bone degradation and inhibit bone formation. Indeed, the degree of the inflammation correlates with the extent of local and systemic bone loss [5, 6]. Unfortunately, population-based evidence about pro-inflammatory cytokines and osteoporosis is still rare and too limited to draw conclusions.

In addition, bone mineral density (BMD) is a surrogate indicator of bone strength, which plays an important role in the management of osteoporosis and related fractures. Different from areal BMD measured by dual-energy X-ray absorptiometry (DXA), bone mineral density by quantitative computed tomography (QCT) is a volumetric measure of the vertebral trabecular bone. This approach is convenient for the assessment of BMD during routine abdominal and/or lung CT scans, and for the identification of patients who have an increased risk of fracture with diagnostic CT scanning. Given the high turnover rate of trabecular bone compared to cortical bone, BMD calculated from QCT 
offers substantially higher sensitivity [7, 8]. However, studies of osteoporosis using QCT are far fewer than research projects using DXA, considering that QCT is a newly developed technic.

Therefore, in this cross-sectional study, we used the QCT-measured BMD and the levels of related inflammatory cytokines to explore their gender-specific association, based on a middle-aged and older population in Beijing, China.

\section{Material and methods}

\section{Study population}

The participants of this study were a subset of the PURE CASH study population in the Beijing Jishuitan hospital. The subjects of the PURE CASH study and study protocol were reported previously (QIMS paper) [8, 9]. During the fourth follow-up visit, blood samples were collected and QCT scans were performed. The study protocol was approved by the Ethics Committee of Beijing Jishuitan hospital.

\section{QCT measurement}

As part of the protocols of the PURE CASH China study, all subjects underwent abdominal QCT scans. All QCT scans and image analyses were performed in the department of Radiology, Beijing Jishuitan Hospital. The details of the QCT acquisition were reported previously [8]. Briefly, a standard abdominal CT scan was performed for each of the subjects on a Toshiba 64-slice or on an 80-slice CT scanner with a Mindways QCT pro (Mindways, Austin, TX, USA) calibration phantom positioned under the back. The BMD of L1-3 lumbar vertebral body was measured with the Mindways QCT pro v5 software according to the manufactory's instruction.

\section{Adipose tissue measurement}

The CT volumetric dataset was transferred to a Mindways QCT workstation and the measurement of abdominal adipose tissue was performed by trained and qualified radiologists. The details of adipose tissue measurement of each slice were described in earlier publications [9]. Six slices at each intervertebral space from T12 / L1 to L5/S1, as well as an extra slice at the umbilicus level, were measured. Total adipose tissue (TAT) and visceral adipose tissue (VAT) area $\left(\mathrm{cm}^{2}\right)$ were semi-automatically measured by the tissue composition module of the software (Mindways, Austin, TX, USA).

\section{Plasma measurement and other data collection}

All physical examinations (blood pressure, height, and weight) and sample tests were measured according to a standardised protocol and conducted by trained physicians. Tumour necrosis factor $\alpha(\mathrm{TNF}-\alpha)$, interleukin $1 \beta$ (IL-1 $\beta)$, IL-6, IL-17, human monocyte chemoattractant protein-1 (MCP-1), and plasminogen activator inhibitor-1 (PAI-1) were measured by a multi-factor detection instrument (MACPIX, LUMINEX) using HADCYMAG-61K MILLIPLEX MAP Human Adipocyte Magnetic Bead Panel-Endocrine Multiplex Assay. The fully automatic multifunctional enzyme labelling instrument used was a MULTISKAN MK3 (Thermo, USA).

\section{Definitions of osteoporosis and BMI}

Participants were categorised into three groups according the measurement of BMD: (1) normal bone mass: BMD $>120 \mathrm{mg} / \mathrm{cm}^{3}$, (2) osteopaenia: BMD $80-120 \mathrm{mg} / \mathrm{cm}^{3}$, and (3) osteoporosis: BMD $<80 \mathrm{mg} / \mathrm{cm}^{3}[10,11]$. Body mass index (BMI) was calculated as weight $(\mathrm{kg})$ divided by height squared $\left(\mathrm{m}^{2}\right)$.

\section{Statistical analysis}

Data were expressed as mean \pm SD for continuous variables and percentage $(\%)$ for categorical variables. The significant difference in continuous and categorical variables was tested by a oneway ANOVA and a chi-square test, respectively, comparing the distribution of three groups of BMD. A non-parametric test was performed if the model of the ANOVA was not satisfied. Multivariable ordinal logistic regression was used to assess the association of gender-specific plasma pro-inflammatory cytokines and three groups of $\mathrm{BMD}$, and the odds ratio (OR) and $95 \%$ confidence intervals (CIs) were calculated. All analyses were conducted using SPSS 19.0 for windows (serial number: 5076595) after being double entered. A two-sided P value $<0.05$ was considered statistically significant.

\section{Results}

A total of 85 subjects with complete inflammation factors and complete QCT data were included in our analysis, with $40.00 \%$ (34) being men. The mean age was $63.24 \pm 7.4$ years (48-85 years). BMD ranged from 24.30 to 204.15, and the proportions of osteoporosis, osteopaenia, and normal BMD were $29.41 \%$ (25), 37.65\% (32), and $32.94 \%$ (28), respectively. Gender-specific distribution of each BMD group is presented in Figure 1. The distribution of BMD varied in gender $(\mathrm{p}<0.01)$. Males had the highest proportion of osteopaenia (47.06\%), while females had osteoporosis (39.22\%).

\section{Clinical features of each BMD group}

The general condition and all tested clinical features of participants in the three BMD groups were compared (see Tab. I). Participants with osteoporosis had the lowest test values in CRE ( $p=0.004)$ and the oldest age $(p<0.001)$. U-TAT and TP had a decreasing trend, while GLU and TG increased gradually from normal BMD to osteoporosis, but no significant statistical difference was found $(\mathrm{p}>0.05)$.

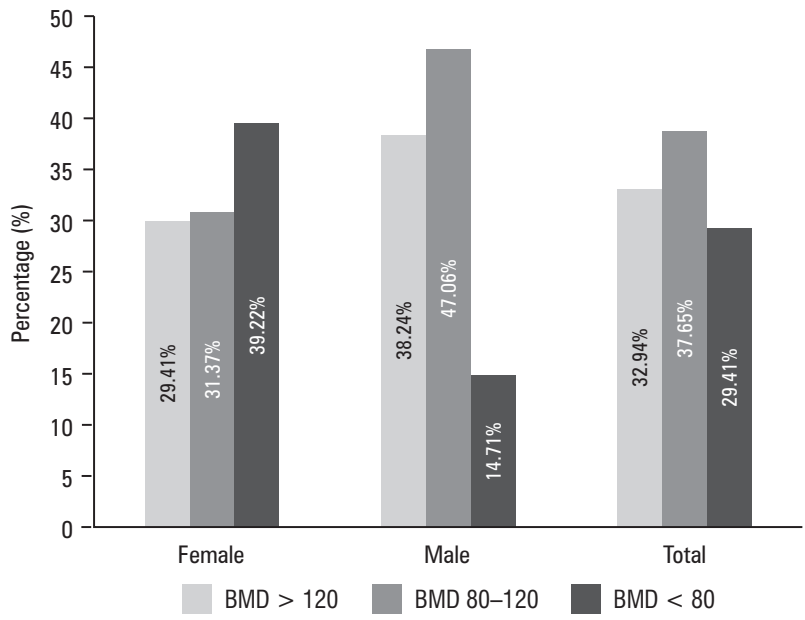

Figure 1. Gender-specific distribution of each BMD group. BMD - bone mineral density 
Table I. Characteristics of the participants in each BMD group

\begin{tabular}{|c|c|c|c|c|c|}
\hline & Normal BMD (n = 28) & Osteopaenia (n = 32) & Osteoporosis (n $=25$ ) & Total $(n=85)$ & p value \\
\hline \multicolumn{6}{|c|}{ General conditions } \\
\hline Age & $59.07 \pm 7.21$ & $63.91 \pm 6.52$ & $67.04 \pm 6.52$ & $63.24 \pm 7.4$ & 0.000 \\
\hline BMI & $25.77 \pm 4.58$ & $26.51 \pm 4.02$ & $25.92 \pm 4.50$ & $26.10 \pm 4.29$ & 0.829 \\
\hline SBP & $132.12 \pm 16.79$ & $139.02 \pm 15.15$ & $139.08 \pm 17.96$ & $136.67 \pm 16.59$ & 0.228 \\
\hline DBP & $77.04 \pm 7.48$ & $76.8 \pm 9.23$ & $76.6 \pm 8.91$ & $76.83 \pm 8.47$ & 0.985 \\
\hline \multicolumn{6}{|c|}{ Pro-inflammatory factors } \\
\hline MCP-1 & $209.4 \pm 74.86$ & $227.17 \pm 65.38$ & $225.31 \pm 86.45$ & $220.77 \pm 74.69$ & 0.619 \\
\hline TNF- $\alpha$ & $2.71 \pm 0.99$ & $2.68 \pm 0.76$ & $2.75 \pm 0.79$ & $2.71 \pm 0.84$ & 0.950 \\
\hline $\mathrm{IL}-1 \beta$ & $1.09 \pm 1.51$ & $1.77 \pm 3.1$ & $0.74 \pm 0.26$ & $1.24 \pm 2.12$ & 0.099 \\
\hline IL-6 & $3.85 \pm 5.94$ & $4.58 \pm 4.06$ & $3.19 \pm 1.14$ & $3.93 \pm 4.26$ & 0.476 \\
\hline IL-17 & $11.05 \pm 17.27$ & $16.43 \pm 32.31$ & $15.71 \pm 21.33$ & $14.45 \pm 24.84$ & 0.676 \\
\hline PAl-1 & $16698.46 \pm 3581.24$ & $14509.47 \pm 3810.76$ & $15131.72 \pm 4026.12$ & $15413.56 \pm 3872.77$ & 0.083 \\
\hline \multicolumn{6}{|c|}{ Adipose volume } \\
\hline $\mathrm{L} 2 / 3 \mathrm{TAT}$ & $329.84 \pm 119.24$ & $339.83 \pm 106.39$ & $328.76 \pm 120.23$ & $333.28 \pm 113.59$ & 0.920 \\
\hline L2/3 VAT & $190.12 \pm 78.47$ & $199.58 \pm 73.43$ & $186.09 \pm 76.97$ & $192.5 \pm 75.46$ & 0.787 \\
\hline U-TAT & $361.82 \pm 133.35$ & $369.32 \pm 103.6$ & $396.22 \pm 118.66$ & $374.76 \pm 117.91$ & 0.545 \\
\hline U-VAT & $165.05 \pm 52.32$ & $162.06 \pm 52.83$ & $162.00 \pm 54.76$ & $163.03 \pm 52.62$ & 0.970 \\
\hline \multicolumn{6}{|c|}{ Regular examinations } \\
\hline ALT & $17.36 \pm 6.97$ & $20.92 \pm 9.98$ & $16.84 \pm 8.69$ & $18.55 \pm 8.8$ & 0.151 \\
\hline TP & $72.26 \pm 4.53$ & $73.78 \pm 4.05$ & $74.62 \pm 3.56$ & $73.53 \pm 4.15$ & 0.105 \\
\hline CRE & $71.04 \pm 16.63$ & $69.66 \pm 10.69$ & $59.52 \pm 12.36$ & $67.13 \pm 14.13$ & 0.004 \\
\hline GLU & $6.65 \pm 3.08$ & $6.30 \pm 2.10$ & $5.96 \pm 1.97$ & $6.32 \pm 2.42$ & 0.586 \\
\hline T-CHO & $5.03 \pm 0.83$ & $4.99 \pm 0.99$ & $4.99 \pm 0.92$ & $5.01 \pm 0.91$ & 0.982 \\
\hline TG & $1.93 \pm 1.29$ & $1.74 \pm 1.15$ & $1.35 \pm 0.61$ & $1.69 \pm 1.09$ & 0.146 \\
\hline
\end{tabular}

$\mathrm{BMD}$ - bone mineral density: normal BMD $=\mathrm{BMD}>120 \mathrm{mg} / \mathrm{cm}^{3} ;$ osteopaenia $=\mathrm{BMD} 80-120 \mathrm{mg} / \mathrm{cm}^{3} ;$ osteoporosis $=\mathrm{BMD}<80 \mathrm{mg} / \mathrm{cm}^{3} ; \mathrm{BMI}-$ body mass index; SBP — systolic blood pressure; DBP — diastolic blood pressure; MCP-1 — human monocyte chemoattractant protein-1; TNF- $\alpha$ - tumour necrosis factor $\alpha$; IL — interleukin; PAI-1 — plasminogen activator inhibitor-1; L2/3 TAT — L2/3 layer of total adipose tissue; L2/3 VAT — L2/3 layer of visceral adipose tissue; U-TAT — umbilicus level of total adipose tissue; U-VAT — umbilicus level of visceral adipose tissue; ALT — alamine aminotransferase; TP — total protein; CRE — serum creatinine; GLU — fasting blood glucose; T-CHO — total cholesterol; TG — triglyceride

\section{Gender-specific comparison of pro-inflammatory cytokines in different BMD groups}

The distribution of pro-inflammatory cytokines in different BMD groups by gender is shown in Table II. In females, the level of IL- 6 was significantly different in each BMD group ( $\mathrm{p}=0.028)$, while the level of TNF$\alpha$ showed an increasing trend with decreasing BMD $(p=0.063)$. The levels of MCP-1, TNF- $\alpha$, and IL-17 in osteopaenia and osteoporosis were higher than the normal, while IL- $1 \beta$ only increased in the osteopaenia group, but all with no statistical significance. No distinct difference could be observed in males.

The gender-difference of pro-inflammatory cytokines in each BMD group was also compared. Males in the normal BMD group had clearly higher TNF- $\alpha$, IL- $1 \beta$, and IL-6 levels than the females ( $\mathrm{p}<0.05)$. And in the osteoporosis group, IL-17 levels were also higher in males $(\mathrm{p}<0.05)$.

\section{ORs of pro-inflammatory cytokines and different BMD groups}

The ORs for increasing levels of pro-inflammatory cytokines with decreasing BMD group are shown in Table III. The findings did not vary remarkably when all participants were analysed.

In contrast to the null findings of the males, a significant statistical difference was observed in females after further adjustment by gender and sex hormones in Model 2. Compared to the normal BMD group, the OR for the decreasing BMD group (osteopaenia and/or osteoporosis) with increasing levels of IL- $1 \beta$ was 0.50 (95\% CI: 0.25-0.99), and it remained significant ( $\mathrm{OR}=0.03$, 95\% CI: 0.002, 0.042) after all adjustments in Model 3. Meanwhile, an apparent association was found in IL-6, PAI-1, and MCP-1 in females after all adjustment; the adjusted ORs were 9.50 (95\% CI: 1.44-62.80), 1.001 (95\% CI: 1.000-1.002), and 0.97 (95\% CI: 0.94-1.00), respec- 
Table II. Gender-specific comparison of pro-inflammatory cytokines in each BMD group

\begin{tabular}{lccccc}
\hline & Normal BMD $(\mathbf{n}=\mathbf{2 8})$ & Osteopaenia $(\mathbf{n}=\mathbf{3 2})$ & Osteoporosis $(\mathbf{n}=\mathbf{2 5})$ & Total $(\mathbf{n}=\mathbf{8 5})$ & $\mathbf{p}$ value \\
\hline Female & & & & & \\
\hline MCP-1 & $201.08 \pm 79.54$ & $229.34 \pm 82.27$ & $226.64 \pm 95.37$ & $219.97 \pm 86.09$ & 0.606 \\
\hline TNF- $\alpha$ & $2.36 \pm 0.65$ & $2.80 \pm 0.61$ & $2.89 \pm 0.72$ & $2.71 \pm 0.69$ & 0.063 \\
\hline IL-1 $\beta$ & $0.65 \pm 0.17$ & $1.94 \pm 3.97$ & $0.67 \pm 0.11$ & $1.06 \pm 2.26$ & 0.403 \\
\hline IL-6 & $2.35 \pm 1.26$ & $5.39 \pm 5.2$ & $3.27 \pm 1.15$ & $3.66 \pm 3.26$ & 0.028 \\
\hline IL-17 & $7.36 \pm 3.03$ & $14.2 \pm 17.69$ & $15.3 \pm 23.92$ & $12.62 \pm 18.05$ & 0.407 \\
\hline PAl-1 & $16675.93 \pm 3693.27$ & $14165.25 \pm 3492.85$ & $15221.25 \pm 3914.76$ & $15317.8 \pm 3779.56$ & 0.181 \\
\hline Male & & & & & \\
\hline MCP-1 & $218.99 \pm 71.01$ & $225.00 \pm 45.32$ & $220.01 \pm 39.94$ & $221.97 \pm 54.49$ & 0.956 \\
\hline TNF-a & $3.11 \pm 1.17^{*}$ & $2.56 \pm 0.89$ & $2.2 \pm 0.89$ & $2.72 \pm 1.03$ & 0.170 \\
\hline IL-1b & $1.61 \pm 2.13^{* *}$ & $1.60 \pm 2.02$ & $0.98 \pm 0.51$ & $1.51 \pm 1.89^{*}$ & 0.805 \\
\hline IL-6 & $5.59 \pm 8.45^{*}$ & $3.76 \pm 2.36$ & $2.88 \pm 1.18$ & $4.33 \pm 5.45$ & 0.559 \\
\hline IL-17 & $15.32 \pm 24.97$ & $18.65 \pm 42.83$ & $17.37 \pm 3.05^{*}$ & $17.19 \pm 32.62$ & 0.965 \\
\hline PAI-1 & $16724.46 \pm 3597.49$ & $14853.69 \pm 4190.36$ & $14773.6 \pm 4925.62$ & $15557.21 \pm 4061.79$ & 0.432 \\
\hline
\end{tabular}

Normal BMD = BMD $>120 \mathrm{mg} / \mathrm{cm}^{3} ;$ osteopaenia = BMD $80-120 \mathrm{mg} / \mathrm{cm}^{3} ;$ osteoporosis = BMD < $80 \mathrm{mg} / \mathrm{cm}^{3} ;$ BMI — body mass index; SBP — systolic blood pressure; DBP — diastolic blood pressure; MCP-1 — human monocyte chemoattractant protein-1; TNF- $\alpha$ — tumour necrosis factor $\alpha$; IL — interleukin;

PAI-1 — plasminogen activator inhibitor-1. Male vs. female, ${ }^{*} p<0.05^{* *} p<0.01$

Table III. Sex-specific association of pro-inflammatory cytokines and BMD groups

\begin{tabular}{|c|c|c|c|c|c|c|}
\hline & Female $(n=51)$ & & Male $(n=34)$ & & Total $(n=85)$ & \\
\hline & OR (95\% CI) & $\mathrm{p}$ value & OR (95\% CI) & $\mathrm{p}$ value & OR (95\% CI) & $\mathrm{p}$ value \\
\hline \multicolumn{7}{|c|}{ Model 1} \\
\hline MCP-1 & $1.00(0.99,1.01)$ & 0.659 & $1.01(0.99,1.03)$ & 0.296 & $1.00(1.001 .01)$ & 0.775 \\
\hline TNF- $\alpha$ & $1.95(0.65,5.82)$ & 0.230 & $1.72(0.48,6.22)$ & 0.409 & $1.08(0.57,2.07)$ & 0.806 \\
\hline IL-1 $\beta$ & $0.66(0.35,1.24)$ & 0.198 & $0.57(0.19,1.71)$ & 0.312 & $0.80(0.57,1.13)$ & 0.207 \\
\hline IL-6 & $1.15(0.72,1.80)$ & 0.555 & $0.47(0.20,1.15)$ & 0.098 & $1.01(0.80,1.28)$ & 0.917 \\
\hline IL-17 & $1.03(0.99,1.07)$ & 0.122 & $1.07(0.99,1.15)$ & 0.071 & $1.01(1.00,1.03)$ & 0.137 \\
\hline PAI-1 & $1.00(1.00,1.00)$ & 0.963 & $1.00(1.00,1.00)$ & 0.111 & $1.00(1.00,1.00)$ & 0.173 \\
\hline \multicolumn{7}{|c|}{ Model 2} \\
\hline MCP-1 & $0.99(0.98,1.00)$ & 0.106 & $1.03(0.98,1.08)$ & 0.287 & $1.00(1.00,1.01)$ & 0.748 \\
\hline TNF- $\alpha$ & $3.47(0.95,12.63)$ & 0.059 & $9.98(0.51,196.76)$ & 0.130 & $1.14(0.58,2.24)$ & 0.706 \\
\hline IL-1 $\beta$ & $0.50(0.25,0.99)$ & 0.047 & $0.56(0.12,2.63)$ & 0.465 & $0.82(0.59,1.14)$ & 0.244 \\
\hline IL-6 & $1.52(0.90,2.57)$ & 0.114 & $0.28(0.06,1.37)$ & 0.115 & $1.02(0.85,1.23)$ & 0.835 \\
\hline IL-17 & $1.03(0.99,1.08)$ & 0.117 & $1.09(0.98,1.21)$ & 0.129 & $1.01(0.99,1.03)$ & 0.225 \\
\hline PAI-1 & $1.00(1.00,1.00)$ & 0.323 & $1.00(1.00,1.00)$ & 0.092 & $1.00(1.00,1.00)$ & 0.217 \\
\hline \multicolumn{7}{|c|}{ Model 3} \\
\hline MCP-1 & $0.97(0.94,1.00)$ & 0.031 & $1.07(0.01,82.85)$ & 0.976 & $1.00(1.00,1.01)$ & 0.309 \\
\hline TNF- $\alpha$ & $32.27(0.67,1560.87)$ & 0.079 & $\begin{array}{c}1.44 E+09 \\
(0,3.54 E+40)\end{array}$ & 0.568 & $1.15(0.52,2.54)$ & 0.729 \\
\hline IL-1 $\beta$ & $0.03(0.002,0.042)$ & 0.010 & $0(0,3.84 \mathrm{E}+57)$ & 0.921 & $0.70(0.49,1.01)$ & 0.053 \\
\hline IL-6 & $9.50(1.44,62.80)$ & 0.019 & $0.02(0,8.74 \mathrm{E}+07)$ & 0.739 & $1.07(0.88,1.30)$ & 0.484 \\
\hline IL-17 & $1.10(1.00,1.22)$ & 0.054 & $1.37(0.01,145.91)$ & 0.895 & $1.02(1.00,1.04)$ & 0.100 \\
\hline PAI-1 & $1.001(1.000,1.002)$ & 0.024 & $1.00(0.97,1.03)$ & 0.920 & $1.00(1.00,1.00)$ & 0.291 \\
\hline
\end{tabular}

Model 1 adjusted for age and regular examinations. Model 2 adjusted for Model 1, sex and sex hormones. Model 3 adjusted for Model 2, adipocytokines and adipose volume. All based on normal BMD group. BMD — bone mineral density; $\mathrm{OR}$ — odds ratio; $\mathrm{Cl}$ — confidence interval 
tively. TNF- $\alpha$ and IL-17 tended to show an increased risk $(\mathrm{OR}>1)$ but did not reach significance.

\section{Discussion}

To the best of our knowledge, our study is the first to use QCT to demonstrate the sex-specific relationship between pro-inflammatory cytokines and BMD in a Chinese population. We found that IL-6 is a risk factor for osteoporosis in the Chinese female population. And sex hormones and visceral obesity are important confounding factors.

Osteoporosis is one of the most common inflammatory bone-loss conditions, and it has been estimated that more than $50 \%$ of women and $30 \%$ of men over the age of 50 years are susceptible to fractures and bone loss [12]. In our study, participants with osteoporosis were characterised by older age, higher blood pressure, and more metabolic abnormalities. The lack of male osteoporosis might because of obviously higher prevalence osteoporosis in females, especially postmenopausal female deficiency of gonadal function. Clearly, it was essential to stratify the analysis by sex and adjust the effect of age.

Many basic studies have concerned the relationship between bone and immune systems, and been called "osteoimmunology", since the early 1970s [1]. Pro-inflammatory cytokines, which are secreted mainly by macrophages, synovial fibroblasts, and lymphocytes, break the balance of bone homeostasis, typically resulting in a bone loss and erosion [4]. IL- 6 and TNF- $\alpha$ inhibit bone-forming osteoblast cells, and TNF- $\alpha$, IL-6, M-CSF, and MCP-1 can contribute to osteoclast recruitment, maturation, and inhibit osteoclast apoptosis [13]. Shan-Shan Rao et al. found that magnesium silicate-treated mice exhibited a significant increase in the expression of various pro-inflammatory cytokines (including IL-1 $\beta$, IL-6, and TNF- $\alpha$ ) and accumulation of pro-inflammatory macrophages in the bone marrow [6]. IL-6, IL-17, and IL-23 were considered to play a key role in the pathogenesis of obesity-related osteoarthritis [14].

The present study is the first to use QCT to provide evidence that each unit increase in level of IL- 6 was associated with a nine-fold higher risk of low bone mass and osteoporosis in females, independent of age, sex hormones, adipocytokines, and other potentially confounding factors. The level of IL-6 is extremely low in healthy people and gradually increases with increasing of age or menopause [15]. IL-6 can affect the early stage of osteoclasts, stimulate the proliferation and division of osteoclast precursors, increase the number of osteoclasts, and promote the formation of osteoclast pits. It can also increase the release of collagenase to promote matrix degradation and affect osteoblast activity [16].
In a clinical study, Yan L et al. reported that the GG genotype of the $-634 \mathrm{C} / \mathrm{G}$ polymorphism in IL-6 appears to play a role in reducing BMD, which affects normal bone metabolism and leads to osteoporosis [17].

The current OR results cannot confirm the relationships of IL- $1 \beta$, which sit on the opposite ends of existing biological mechanisms. Evidence has shown the negative effects of IL- $1 \beta$ on the occurrence and development of osteoporosis [18]. Chen HY et al. reported that the Taq I IL-1 $\beta$ exon 5 gene polymorphism is associated with reduced BMD and predisposes women to osteoporosis at the lumbar spine [19]. The compound effects between IL- $1 \beta$ and sex hormones and metabolism might be another reason. As a result, even though the $P$ value is statistically significant, practical value and reliability are limited from a clinical perspective, and more population-based studies are needed to prove this relationship.

Current basic studies have also shown that IL-17 enhances osteoclastogenesis by stimulating osteocytes and osteoblasts, and it potentiates osteoclastogenic activity via upregulation of receptor activator of NF- $\kappa$ B (RANK) production to produce higher levels of RANKL [20]. In our analysis, IL-17 tended to show an increased risk $(\mathrm{OR}>1)$, but without statistical significance owing to the limited sample size. Similar happens to TNF- $\alpha$. The ORs of PAI- 1 and MCP-1 are too close to 1 to determine the practical meaning. All poor practice and statistic of these pro-inflammatory cytokines might due to the complicated mutual effects. For example, Lacativa P et al. revealed that IL- $1 \beta$ has an intimate relationship with TNF- $\alpha$, and many effects of TNF- $\alpha$ are upregulated by IL-1 $\beta$ during inflammatory osteoclastogenesis [21]. Rosuvastatin could decrease both spontaneous and IL-1 $\beta$-induced IL-6 mRNA expression in osteoblasts [22].

In our gradually modified logistic models, no significance presented when only the effect of age and regular examinations were controlled. Obviously, significant statistics appeared after further adjustment of sex hormone, adipocytokines and adipose volume. In other words, osteoimmunology-related researches need to pay attention to the confounding effects of sex hormones and fat-related factors in case of false-negative results. Current available studies have revealed their influence and underlying mechanisms. A study surveying 455 participants with menopause showed that women with osteoporosis had lower levels of oestrogen and higher levels of oxidative stress, when compared with non-osteoporosis subjects [23]. Once there was a lack of oestrogen, monocyte-produced TNF- $\alpha$ and lymphocyte-produced TNF- $\beta$ would rapidly increase, indirectly activating the mature of osteoclasts and other cytokines like 1L-6 to promote proliferation of osteoclast precursors [24]. Obesity 
is also correlated with inflammation. Adipose tissue can release pro-inflammatory cytokines like TNF- $\alpha$ and IL- 6 , and, through the signals of TGF- $\beta$ or RANK/RANKL to facilitate the activation and differentiation of osteoclast, inhibit osteoclast apoptosis, and promote osteogenesis absorption [25].

Several limitations in this study need to be considered. First, the temporal and casual relationship could not be assessed in a cross-sectional design. Second, although multiple variables were adjusted, other confounding factors, such as alcohol intake, menopausal age, lifestyle habits, and recent usage of medicines, may exist. Third, the changes in pro-inflammatory mediator levels in blood are not as obvious as in bone; only the serum concentration of IL- 6 was found to be marked risk factor. Also, one of the reasons may be the small sample size. Finally, population-based links between inflammatory factors and osteoporosis are still not fully elucidated. Long-term and large-scale population-based studies are therefore needed to reveal such associations and to explore the role of the immune system in osteoporosis.

\section{Conclusions}

IL-6 is an independent risk factor of osteoporosis measured by QCT in middle-aged and older females in China. Sex hormones and fat-related factors are important confounders in the research of osteoimmunology.

\section{Acknowledgments}

We thankfully acknowledge all the participants in this study for sharing their time. We also warmly thank all the laboratory staff for their excellent assistance in performing measurements.

\section{Author contribution}

The first authorship of J.Z. and K.L. is of an equal rank. J.Z. and K.L. contributed to the analysis and interpretation of the data, and they drafted the manuscript. Y.P.G. and X.G.C. designed and gave guidance on writing this paper. C.L.L., L.X., W.L., and Y.Z. contributed to the acquisition and interpretation of the data. All authors have read and approved the final manuscript.

\section{Conflict of interest}

The authors declare no conflict of interest.

\section{Funding}

This research was funded by grants from the Military Logistics Research Projects of China (No. CWS13J054) and the Translational Medicine Projects of the People's Liberation Army General Hospital (No. 2017TM-021).

\section{References}

1. Srivastava RK, Dar HY, Mishra PK. Immunoporosis: Immunology of Osteoporosis-Role of T Cells. Front Immunol. 2018; 9: 657, doi: 10.3389/fimmu.2018.00657, indexed in Pubmed: 29675022

2. China health promotion foundation of osteoporosis prevention and control. Osteoporosis in China. Chinese J Health Manage. 2009; 3(3): 148-153, doi: 10.3760/cma.j.issn.1674-0815.2009.03.006.

3. Si L, Winzenberg TM, Jiang Q, et al. Projection of osteoporosis-related fractures and costs in China: 2010-2050. Osteoporos Int. 2015; 26(7): 1929-1937, doi: 10.1007/s00198-015-3093-2, indexed in Pubmed: 25761729.

4. Franceschi C, Campisi J. Chronic inflammation (inflammaging) and its potential contribution to age-associated diseases. J Gerontol A Biol Sci Med Sci. 2014; 69 Suppl 1: S4-S9, doi: 10.1093/gerona/glu057, indexed in Pubmed: 24833586.

5. Amarasekara DS, Yu J, Rho J. Bone Loss Triggered by the Cytokine Network in Inflammatory Autoimmune Diseases. J Immunol Res. 2015; 2015: 832127, doi: 10.1155/2015/832127, indexed in Pubmed: 26065006.

6. Rao SS, Hu Y, Xie PL, et al. Omentin-1 prevents inflammation-induced osteoporosis by downregulating the pro-inflammatory cytokines. Bone Res. 2018; 6: 9, doi: 10.1038/s41413-018-0012-0, indexed in Pubmed: 29619269.

7. Pickhardt PJ, Bodeen G, Brett A, et al. Comparison of femoral neck BMD evaluation obtained using Lunar DXA and QCT with asynchronous calibration from CT colonography. J Clin Densitom. 2015; 18(1): 5-12, doi: 10.1016/j.jocd.2014.03.002, indexed in Pubmed: 24880495.

8. Wang L, Su Y, Wang Q, et al. Validation of asynchronous quantitative bone densitometry of the spine: Accuracy, short-term reproducibility, and a comparison with conventional quantitative computed tomography. Sci Rep. 2017; 7(1): 6284, doi: 10.1038/s41598-017-06608-y, indexed in Pubmed: 28740145.

9. Cheng X, Zhang Y, Wang C, et al. The optimal anatomic site for a single slice to estimate the total volume of visceral adipose tissue by using the quantitative computed tomography (QCT) in Chinese population. Eur J Clin Nutr. 2018; 72(11): 1567-1575, doi: 10.1038/s41430-018-0122-1, indexed in Pubmed: 29559725.

10. American College of Radiology 2008. ACR-SPR-SSR Practice Parameter for the Performance of Quantitative Computed Tomography (QCT) Bone Densitometry (Amended 2014 Resolution 39). Reston, Va, USA. http:// www.acr.org/ /media/ACR/Documents/PGTS/guidelines/QCT.pdf..

11. Ma XH, Zhang W, Wang Y, et al. Comparison of the Spine and Hip BMD Assessments Derived from Quantitative Computed Tomography. In J Endocrinol. 2015; 2015: 675340, doi: 10.1155/2015/675340, indexed in Pubmed: 26273301

12. Eisman JA, Bogoch ER, Dell R, et al. ASBMR Task Force on Secondary Fracture Prevention. Making the first fracture the last fracture: ASBMR task force report on secondary fracture prevention. J Bone Miner Res. 2012; 27(10): 2039-2046, doi: 10.1002/jbmr.1698, indexed in Pubmed: 22836222.

13. Collins KH, Herzog W, MacDonald GZ, et al. Obesity, Metabolic Syndrome, and Musculoskeletal Disease: Common Inflammatory Pathways Suggest a Central Role for Loss of Muscle Integrity. Front Physiol. 2018; 9: 112, doi: 10.3389/fphys.2018.00112, indexed in Pubmed: 29527173.

14. Iannone F, Lapadula G. Obesity and inflammation - targets for OA therapy. Curr Drug Targets. 2010; 11(5): 586-598, indexed in Pubmed: 20199391.

15. Eftekhari H, Hosseini S, Baboli HP, et al. Association of interleukin-6 (rs1800796) but not transforming growth factor beta 1 (rs1800469) with serum calcium levels in osteoporotic patients. Gene. 2018; 671: 21-27, doi: 10.1016/j.gene.2018.05.118, indexed in Pubmed: 29860063

16. Krela-Kaźmierczak I, Szymczak-Tomczak A, Łykowska-Szuber L, et al. Interleukin 6, osteoprotegerin, sRANKL and bone metabolism in inflammatory bowel diseases. Adv Clin Exp Med. 2018; 27(4): 449-453, doi: 10.17219/acem/75675, indexed in Pubmed: 29558031.

17. Yan L, Hu R, Tu S, et al. Meta-analysis of association between IL-6 $-634 \mathrm{C} / \mathrm{G}$ polymorphism and osteoporosis. Genet Mol Res. 2015; 14(4): 19225-19232, doi: 10.4238/2015.December.29.32, indexed in Pubmed: 26782575.

18. Ruscitti P, Cipriani P, Carubbi F, et al. The role of IL-1 in the bone loss during rheumatic diseases. Mediators Inflamm. 2015; 2015: 782382, doi: 10.1155/2015/782382, indexed in Pubmed: 25954061.

19. Chen HY, Chen WC, Wu MC, et al. Interleukin-1beta and interleukin-1 receptor antagonist gene polymorphism in postmenopausal women: correlation to bone mineral density and susceptibility to osteoporosis. Maturitas. 2003; 44(1): 49-54, indexed in Pubmed: 12568735.

20. Kocić J, Santibañez JF, Krstić A, et al. Interleukin 17 inhibits myogenic and promotes osteogenic differentiation of $\mathrm{C} 2 \mathrm{C} 12$ myoblasts by activating ERK1,2. Biochim Biophys Acta. 2012; 1823(4): 838-849, doi: 10.1016/j. bbamcr.2012.01.001, indexed in Pubmed: 22285818.

21. Lacativa PG, Farias ML. Osteoporosis and inflammation. Arq Bras Endocrinol Metabol. 2010; 54(2): 123-132, indexed in Pubmed: 20485900. 
22. Lazzerini PE, Capperucci C, Spreafico A, et al. Rosuvastatin inhibits spontaneous and IL- $1 \beta$-induced interleukin- 6 production from human cultured osteoblastic cells. Joint Bone Spine. 2013; 80(2): 195-200, doi: 10.1016/j.jbspin.2012.07.007, indexed in Pubmed: 22999910.

23. D'Amelio P, Fornelli G, Roato I, et al. Interactions between the immune system and bone. World J Orthop. 2011; 2(3): 25-30, doi: 10.5312/wjo. v2.i3.25, indexed in Pubmed: 22474632
24. Kimble RB, Bain S, Pacifici R. The functional block of TNF but not of IL-6 prevents bone loss in ovariectomized mice. J Bone Miner Res. 1997; 12(6) 935-941, doi: 10.1359/jbmr.1997.12.6.935, indexed in Pubmed: 9169353.

25. Luo XH, Guo LJ, Xie H, et al. Adiponectin stimulates RANKL and inhibit OPG expression in human osteoblasts through the MAPK signaling pathway. J Bone Miner Res. 2006; 21(10): 1648-1656, doi: 10.1359/jbmr.060707, indexed in Pubmed: 16995820. 